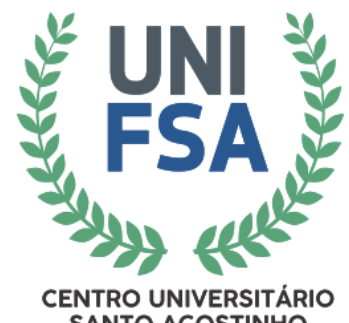

SANTO ACOSTINHO

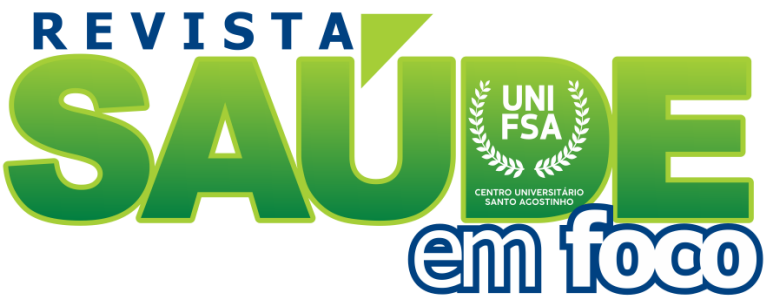

www4.fsanet.com.br/revista

Revista Saúde em Foco, Teresina, v. 8, n. 2, art. 1, p. 03-14, mai./ago. 2021

ISSN Eletrônico: 2358-7946

http://dx.doi.org/10.12819/rsf.2021.8.2.1

\title{
Descrição dos Níveis de Autoestima, Autoimagem, Resiliência e Felicidade de Estudantes Adolescentes
}

\author{
Description of the Levels of Self-Esteem, Self-Image, Resilience and Happiness of Adolescent \\ Students
}

Felipe Ferreira de Sousa

Graduação em Psicologia-UNIFIP

Vínculo institucional: Universidade Federal de Campina Grande

E-mail: felipeufcg19.2@gmail.com

Renata Andrea Salvitti de Sá Rocha

Pós-Doutorado em Psicologia Aplicada à Odontologia - FOP Unicamp Doutora em Odontopediatria - área de Psicologia Aplicada à Odontologia - FOP Unicamp E-mail: renatasarocha@hotmail.com

Endereço: Felipe Ferreira de Sousa

Avenida Universitária, s/n · Bairro Santa Cecília Patos/PB. CEP: 58708-110, Brasil.

Endereço: Renata Andrea Salvitti de Sá Rocha CSTR UFCG Av. Universitária s/n Bairro Santa Cecília Patos PB, Brasil.
Editor-Chefe: Dr. Tonny Kerley de Alencar Rodrigues

Artigo recebido em 17/10/2021. Última versão recebida em 28/10/2021. Aprovado em 29/10/2021.

Avaliado pelo sistema Triple Review: a) Desk Review pelo Editor-Chefe; e b) Double Blind Review (avaliação cega por dois avaliadores da área).

Revisão: Gramatical, Normativa e de Formatação 


\title{
RESUMO
}

O presente Projeto de TCC tem como objetivo geral analisar as relações existentes entre autoestima, autoimagem, resiliência e felicidade entre estudantes adolescentes em uma cidade do sertão da Paraíba. Para tanto, a pesquisa será de campo, com natureza quantitativadescritiva, com corte transversal e amostral e observação direta extensiva (realizada através de questionário), por meio de uma amostragem não probabilística. Assim, participaram deste estudo 200 estudantes adolescentes, sendo 100 de escolas públicas e 100 oriundos de escolas privadas do município de Patos-PB, com idades variando entre 11 e 19 anos, sendo a maioria 75\% do sexo feminino. Estes responderam a um instrumento composto pelas escalas de Autoestima, Escala de Autoimagem, Escala de Resiliência e Escala de Felicidade Subjetiva. Os dados foram analisados por meio do SPSS-WIN-versão 21.0 - através de estatísticas descritivas e inferenciais, e os resultados discutidos à luz da literatura pertinente. Todos os preceitos éticos foram adotados quanto aos critérios das pesquisas com seres humanos. Os principais resultados revelaram que não foram encontradas correlações significativas entre nenhum dos construtos estudados. Verificou-se que os adolescentes apresentaram diferenças significativas entre os sexos $(t=0,87, g l=200, \mathrm{p}<0,005)$, em que as mulheres $(\mathrm{M}=4,71)$ obtiveram médias maiores que os homens $(\mathrm{M}=4,35)$ na Escala de Felicidade. Portanto, conclui-se que se torna relevante realizar novos estudos sobre Autoestima, Autoimagem, resiliência e felicidade entre adolescentes escolares.

Palavras-chaves: Adolescência. Autoestima. Autoimagem. Resiliência. Felicidade.

\begin{abstract}
The purpose of the present CBT Project is to analyze the relationships between self-esteem, self-image, resilience and happiness among teenage students in a city in the interior of Paraíba. To do so, the research will be field, with quantitative-descriptive nature, crosssection and sample and extensive direct observation (conducted through a questionnaire), through a non-probabilistic sampling. Thus, 200 adolescents participated in this study, of which 100 were from public schools and 100 from private schools in the city of Patos-PB, with ages varying from 11 to 19 years, with a majority being $75 \%$ female. These will respond to an instrument composed of the Self-esteem Scale, Self-Scale Scale, Resilience Scale, and Subjective Happiness Scale. The data were analyzed through SPSS-WIN-version 21.0 through descriptive and inferential statistics, and the results discussed in light of the relevant literature. All the ethical precepts were adopted regarding the criteria of the researches with human beings. The main results showed that no significant correlations were found between any of the constructs studied. It was verified that the adolescents presented significant differences between the sexes $(\mathrm{t}=0.87, \mathrm{gl}=200, \mathrm{p}<0.005)$, where women $(\mathrm{M}=4.71)$ obtained averages larger than males $(M=4.35)$ on the Happiness Scale. Therefore, it is concluded that it becomes relevant to carry out new studies on self-esteem, self-image, resilience and happiness among school adolescents.
\end{abstract}

Keywords: Adolescence. Self Esteem. Self Image. Resilience. Happiness. 


\section{INTRODUÇÃO}

A adolescência é concebida como uma categoria social do tipo geracional por meio da qual se revelam as possibilidades e os constrangimentos da estrutura social. Na Psicologia, conhecer as representações, vivências e construtos no contexto da adolescência torna-se um tema relevante, pois permite apreender diferentes aspectos da sociedade e seus atores. Deve ser considerada como uma condição própria dos sujeitos adolescentes, um conjunto de experiências vividas por eles em diferentes lugares históricos, geográficos e sociais, sendo, portanto, muito mais do que uma representação feita por adultos sobre esta fase da vida (MOSELLI, 2021; GRANOT, 2021.).

Não se chegou a um acordo relativo à adolescência, ainda não foi definido se seu início ocorre antes, durante ou após a puberdade, mas a maior parte dos estudos adotam a puberdade como fator de início. As mudanças corporais que ocorrem nessa fase do desenvolvimento, tanto nas garotas quando nos meninos, são relevantes. Os hormônios são os principais responsáveis pelas mudanças ocorridas nesse período de vida, que se inicia por volta dos oito ou nove anos, impulsionando mudanças no corpo, no referente às proporções corporais e o desenvolvimento biopsicossocial, atributos sexuais primários e secundários (SAWYER, 2018).

A Organização Mundial da Saúde (OMS) define a adolescência como a intermitência de tempo correspondente à faixa etária que vai, cronologicamente, dos dez aos dezenove anos. Não obstante essa definição, os eventos ocorridos no período conhecido como puberdade podem ocorrer em sujeitos considerados normais do mesmo sexo ou de sexo diferente, com idades diferentes, o que também nos leva a indagar o destaque dado a aspecto cronológico (BLAKEMORE, 2019).

A adolescência é marcada por grandes mudanças, transformações abrangendo os campos biológico, social e emocional. É nesse momento que o sujeito passa a incorporar novos comportamentos, assim como novas práticas, pois esse período é marcado pela busca da autonomia e por uma maior exposição às situações do dia a dia. É neste momento da vida em que a maioria das mudanças ocorrem e acabam implicando em grandes alterações na aparência e que vêm a influenciar na satisfação corporal (BLAKEMORE, 2019).

No referente à Autoimagem, ela é entendida como sendo uma representação polimorfa, mutável, que é capaz de refletir desejos, atitudes emocionais, assim como interações do indivíduo com outros sujeitos. A autoimagem foi definida como "percepção subjetiva total de si mesmo, incluindo a imagem corporal e as impressões da personalidade, 
bem como suas capacidades, e assim por diante (outro termo para autoconceito) (MAY, 2021; HUA,2020; TARRÍO, 2020).

Já a Autoestima é um apontador do bem-estar mental, podendo ser compreendida como o conjunto de ideias e/ou atitudes que cada indivíduo tem sobre si. A autoestima está relacionada às metas de desempenho a serem cumpridas, ela sugere que as pessoas se tornam orgulhosas do que denominou como "sucessos fáceis", e quando comparados os sucessos obtidos por si com os das outras pessoas, desta forma são capazes de demonstrar às outras pessoas que são competentes mesmo quando enfrentam um possível fracasso (RUBIO, 2021, RUBIO,2021).

Sendo indispensável dizer que a felicidade integra uma manifestação superior ao que se entende por ou como sendo qualidade de vida, assim como ao bem-estar enquanto característica estabilizadora que lhe é intrínseca. Para estes autores, a felicidade é envolta por sentimentos que podem variar entre positivos e negativos. E está relacionada ao comportamento humano, à generosidade (KATSUMI, 2021; BARRAZA MACÍAS, 2020.).

A Resiliência trata-se da capacidade que uma pessoa/adolescente tem de transcender uma situação complicada, conseguindo superar e sair mais forte. E está ligada a variáveis como: habilidades de dar e receber em relações humanas, disciplina, responsabilidade, receptividade e tolerância ao sofrimento. Por isso consideramos estas variáveis importantes para este estudo com adolescentes (MASTEN, 2002; FELDMAN, 2020.).

Diante do exposto, o presente artigo tem por objetivo geral analisar e descrever os níveis de autoestima, autoimagem, resiliência e felicidade entre estudantes adolescentes em uma cidade do sertão da Paraíba. Especificamente.

\section{METODOLOGIA}

O presente estudo configura-se como uma pesquisa descritiva, quanto aos objetivos de campo, com abordagem quantitativa. A pesquisa foi realizada em uma escola pública e em outra escola privada do município de Patos-PB. A amostra do estudo foi composta por 200 estudantes adolescentes, sendo 100 alunos de escolas públicas e 100 alunos de escolas privadas, todos integrantes do sistema de ensino da cidade de Patos-PB. Quanto ao sexo, os 200 adolescentes são divididos entre $25 \%$ do sexo masculino e $75, \%$ do sexo feminino.

Antes da realização da pesquisa, o presente projeto foi submetido ao Comitê Ético, e aprovado. A referida pesquisa foi realizada após autorização da coordenadora e demais 
autoridades responsáveis pelas escolas, campo de pesquisa, levando em consideração os aspectos éticos que envolvem as pesquisas com seres humanos, de acordo com a Resolução $n^{\circ}$ 466/2012 e Resolução nº 510 /2016, expedidas pelo Conselho Nacional de Saúde.

Como instrumento utilizado, destaca-se a "Escala de Resiliência de Connor-Davidson (CD-RISC)", desenvolvida por Connor e Dvidson (2003), que consiste em um questionário de autorrelato com 25 itens de múltipla escolha. Os itens que compõem a CD-RISC-10 avaliam as percepções dos indivíduos da sua capacidade de adaptação à mudança, de superar obstáculos, de dar a volta por cima depois de doenças, lesões ou outras dificuldades. O instrumento é autoaplicado e os participantes registram suas respostas em uma escala de 0 (nunca é verdade) a 4 (sempre é verdade) (LOPES, 2011).

A escala apresenta como fatores: Fator I, nomeado de "competência social", sugere que os indivíduos possuem autoconfiança, independência, determinação, invencibilidade, controle, desenvoltura e perseverança. Fator II, nomeado como "aceitação de si mesmo e da vida", sugere que os indivíduos possuem adaptabilidade, equilíbrio, flexibilidade e perspectiva de vida equilibrada. Fator III, independência e determinação, sugere que o indivíduo está desassociado das antigas relações de que era totalmente dependente ou era dominado. Determinação sugere que é a ação de tomar decisões/resoluções de maneira fixa.

A "Escala de Felicidade Subjetiva" foi originalmente designada de Subjective Happiness Scale (SHS), desenvolvida por Lyubomirsky (1999), com o objetivo de avaliar a felicidade, foi traduzida por Bertoquini e Pais-Ribeiro (2004). A escala é composta por quatro itens, todos formulados numa direção positiva e com formato de resposta do tipo Likert que varia entre 1 a 7 pontos, 1 (Não muito feliz) e 7 (Muito feliz). Os itens desta escala avaliam a componente cognitiva do bem-estar subjetivo, a satisfação com a vida. Dois itens pedem aos sujeitos para se caracterizar a si próprio, primeiro como caracterização absoluta e depois por comparação aos outros. Os outros dois itens, referem-se a breves descrições de pessoas felizes e de pessoas infelizes, pedindo para o sujeito verificar em que medida se identifica mais ou menos com elas (PAIS-RIBEIRO,2012).

A "Escala de autoestima Rosenberg" (UNIFESP/EPM) trata-se de um instrumento contendo 10 itens, sendo que cinco avaliam sentimentos positivos do humano sobre si mesmo e cinco avaliam sentimentos negativos; cada item é avaliado por uma escala de três pontos, do tipo Likert (concordo fortemente, concordo, discordo e discordo fortemente). O intervalo dessa escala varia de zero a 30; pontuações entre 15 e 25 estão dentro da normalidade, abaixo de 15 sugerem baixa autoestima e acima de 25 elevada autoestima (SBICIGO,2010). 
A "Escala de Autoimagem foi desenvolvida por Singelis (1994), que consiste em um questionário de autorrelato com 24 itens de múltipla escolha. O instrumento é autoaplicado e os participantes registram suas respostas em uma escala de 0 (nunca é verdade) a 4 (sempre é verdade). Esta escala é do tipo Likert, com os extremos 1 (Discordo Totalmente) e 7 (Concordo Totalmente). Os itens foram aleatoriamente distribuídos com relação às duas dimensões deste instrumento: 1) Autoimagem Dependente: "Gosto de ser único e diferente das outras pessoas em muitos aspectos", "Faço minhas próprias coisas, independentemente do que pensam as outras pessoas" e 2) Autoimagem interdependente: por exemplo, "Sacrificarei meu próprio interesse em benefício do grupo em que estou", "Sinto que meu destino se mistura com o destino daqueles que me rodeiam"(GOUVEIA, 2002).

\section{RESULTADO E DISCUSSÕES}

O artigo objetivou verificar a correlação entre os construtos Autoestima, Autoimagem, Felicidade e Resiliência, porém não foi encontrada nenhuma correlação significativa entre os temas abordados com uma amostra de adolescentes. Em seguida, com o objetivo de verificar se existem diferenças de médias entre homens e mulheres adolescentes nos construtos estudados, foram realizados teste $\mathrm{T}$ de Student. Os principais resultados são descritos a seguir.

Quando realizada a comparação entre adolescentes homens e adolescentes mulheres nos fatores autoimagem interdependente e autoestima, não foi encontrada diferença significativa entre as amostras tanto no fator geral de autoimagem, quanto no de autoestima, sendo a média de autoimagem interdependente dos homens $(M=4,63 ; D P=0,94)$ sendo muito próximo das mulheres que foi de $(M=4,67 ; D P=0,87)$. Já no referente à autoimagem independente dos homens com uma $(M=4,81 ; D P=0,96)$ e as mulheres com $(M=4,64 ; D P$ $=0,89)$. Em comparação com a autoestima geral, os homens obtiveram uma $(M=2,81 ; D P=$ $0,49)$ e as mulheres $(M=2,54 ; D P=0,406)$. Os homens não apresentaram diferença significativa quanto às mulheres em fatores da Escala de autoestima de Rosenberg e a Escala de Autoimagem de Singelis: Autoimagem Interdependente, Autoimagem Independente e Autoestima Geral.

No estudo realizado por Quintero e Zareta (2016), em que comparam a Autoestima com o Desempenho Acadêmico, encontrou-se um nível de correlação e significância que existe entre as variáveis Média de Desempenho Acadêmico e Autoestima. Encontrar uma correlação baixa e não significativa $(r=0,019, \mathrm{p}=0,772)$. Enquanto as dimensões: Amor e 
Amizade (que integram a Escala de Autoestima), correlacionaram-se significativamente com a variável desempenho acadêmico. Desempenho acadêmico e amor $(r=0,136, p=0,042)$ e desempenho acadêmico e amizade $(r=0,180, \mathrm{p}=0,007)$. Não há relação significativa entre o desempenho acadêmico médio e o autoconceito ( $\mathrm{r}=0,047, \mathrm{p}=0,487)$.

Já quando os autores relacionaram a Autoestima com o Autoconceito e Desempenho Acadêmico entre homens e mulheres, que é um construto diferente do estudado neste trabalho, encontraram que as mulheres apresentaram uma média maior de 8,6 pontos (DP 0,7) em comparação aos homens 8,2 (DP 8). Em termos de Autoestima, as mulheres têm uma média de 72,9 (DP 14,8), comparadas aos homens com uma média de 77,6 (SD 14,7). E o autoconceito em mulheres mostra uma média de 71,8 (DP 18,4) contra homens, que resultou em 69,5 (SD 189). Isso indica que, apesar de as mulheres terem melhor desempenho acadêmico médio, elas têm uma autoestima média mais baixa que os homens e, inversamente, uma média maior em termos de autoconceito.

Foi encontrada na pesquisa um trabalho que mais se aproximou desta pesquisa, o de Mosquera e Stobäus (2006) que compararam a Autoimagem, Autoestima e Autorrealização entre universitários, sendo encontradas médias” entre 176,86 (mínimo de 140 e máximo de 222, na Graduação) e 180,71 (mínimo de 138 e máximo de 224, na Pós-Graduação), assim como um índice de Correlação de 0,08 (para nível de significância de 0,05): no teste $t$ de Student, portanto não há diferença significativa nas médias entre a pré e pós-testagem dos alunos".

Além do exposto, também se buscou verificar se adolescentes se diferenciam quanto ao sexo na Escala de Felicidade, e nos três fatores da Escala de Resiliência, são: Competência Social e aceitação de si mesmo, Independência e Determinação e Autoconfiança e capacidade de adaptação a situações que, neste caso, foram encontradas diferenças significativas, os resultados serão expostos a seguir.

Quando realizada a comparação entre homens e mulheres nos fatores felicidade e resiliência, foi encontrada uma relação significativa entre as amostras, tanto no fator geral de felicidade e competência social e aceitação de si mesmo, sendo a média dos homens $(M=$ $4,35 ; D P=1,46)$ e a das mulheres $(M=4,71 ; D P=1,16)$. A média dos homens $(M=2,70$; $D P=0,13)$ também foi encontrada uma relação significativa entre homens e mulheres nos fatores felicidade e independência e determinação das mulheres $(M=2,82 ; D P=, 45)$. E homens $\left(\mathrm{M}=2,70 ; \mathrm{DP}^{`}=0,61\right)$ o mesmo ocorreu no fator Interdependente e determinação $(t$ $(198=2,40, p<0,05)$, sendo a média dos homens $(M=2,85 ; D P=, 49)$ muito próxima da média das mulheres $(M=29,2 ; D P=6,3)$. 
Segundo o estudo realizado por Lopez (2009), que comparou Felicidade, Resiliência e Otimismo entre estudantes de escolas nacionais na cidade de Lima-Peru, foram encontradas diferenças na resiliência média, sendo maior em mulheres em relação aos homens, com um significado estatístico menor que 0,01. De um modo semelhante, foram encontradas diferenças de otimismo em favor das alunas com uma probabilidade menor que 0,001. Enquanto isso e felicidade, não há diferenças entre os dois sexos. Da mesma forma, pode-se observar que, de acordo com as médias obtidas, a amostra apresenta níveis médios de felicidade e níveis médios de resiliência. Em relação ao otimismo, as mulheres apresentam níveis elevados e os homens apresentam baixos níveis. Os dados Felicidade $(\mathrm{M}=22,56$; $\mathrm{DP}=6,12)$ entre homens, entre as mulheres $(\mathrm{M}=22,55 ; \mathrm{DP}=6,45)$, quando comparados os dados relacionados à resiliência obteve-se entre homens $(\mathrm{M}=124,55 ; \mathrm{DP}=18,49)$, mulheres $(\mathrm{M}=130,55 ; \mathrm{DP}=15,57)$.

Apesar da busca ativa de estudos nessa área, em artigos, livros e outras fontes, não foram encontrados estudos específicos que se relacionassem a nenhum dos construtos estudados como Autoestima com a Autoimagem, Autoestima com a Resiliência, Autoestima com a Felicidade Subjetiva, Autoimagem Resiliência, Autoimagem e Felicidade Subjetiva; da mesma forma não foi encontrado nenhum material que comparasse ou realizasse novas combinações destes construtos em participantes adolescentes no Brasil nem no mundo. Iniciamos uma busca ativa de textos no Google acadêmico, restrito aos anos de 2010 a 2017, inserindo os descritores em português autoestima \& autoimagem \& estudantes, sem aspas, sendo encontrados aproximadamente 4,750 resultados. Quando a busca foi feita em outros idiomas, como Espanhol, encontrou-se um resultado aproximado de 3,310. Situação parecida ocorreu quando a busca de texto inseriu os seguintes descritivos em espanhol resiliencia y felicidad subjetiva y estudiantes obtendo um resultado de 5,550, quando buscados termos autoimagem y resiliencia y estudiantes com um resultado de 1,890, com autoestima y resiliencia y estudiantes com resultado de 10,800, com autoimagen y felicidad subjetiva y estudiantes um resultado de 4,230. Similar situação ocorre com os descritores self-image and subjective happiness and students resultado 16,500, self-esteem and resilience and students resultado 19,500, self-image and resilience and students resultado 17,200.

Desse modo, considerando os resultados antes descritos, observa-se a carência de estudos sobre Autoestima, Autoimagem, Resiliência, Felicidade Subjetiva e comparações entre estes fatores entre adolescentes no Brasil e mundialmente. Deixando claro também que a pesquisa interessa nos estudos apenas entre estudantes adolescentes escolares, descartando 
todos os demais estudos realizados fora deste ambiente. Tais discussões revelam a necessidade de estudos sobre esta temática.

\section{CONSIDERAÇÕES FINAIS}

Este estudo teve como objetivo entender a relação entre autoestima e autoconceito e sua relação com a resiliência e a felicidade entre adolescentes escolares; identificar seus sentimentos e emoções implicados nesse processo; verificar as circunstâncias e dificuldades enfrentadas pelas crianças neste processo; e, por fim, discutir sobre a importância da psicologia quanto à utilização de estratégias dentro do ambiente educacional.

Evidenciando que não foram encontradas relações significativas entre nenhum dos construtos estudados. Corroborando com outros estudos que também não encontraram relação significativa entre eles. Espera-se com esta pesquisa trazer algumas contribuições para o contexto acadêmico, educacional e social, permitindo uma discussão sobre a importância da psicologia quanto à utilização de estratégias no enfrentamento dentro do ambiente educacional.

A partir dos estudos da Autoestima, Autoimagem, Resiliência e Felicidade em adolescentes escolares, pode-se afirmar que seus efeitos sobre estas pessoas são constantes e frequentes e que têm importância significativa para receber a devida atenção de pesquisadores dentro e fora do Brasil.

Os profissionais que avaliam, diagnosticam e estão em contato direto com os adolescentes devem tomar o máximo possível de precauções e demonstrar um genuíno comprometimento com estas pessoas. Se faz essencial o estudo destas dimensões e de cada caso de modo individual, assim como outras fontes de informação, como a própria família, os amigos e as instituições escolares onde eles estão inseridos, para que desta forma se tenha uma melhor apuração dos fatos.

Atualmente, é percebido um aumento considerável nos índices de baixa autoestima, comprometimento da autoimagem, baixo nível de felicidade e resiliência entre adolescentes, que por sua vez envolve alguns graus de morbidade, em algumas ocasiões levando ao suicídio, por estes motivos devendo se tornar uma preocupação indispensável pelas redes de saúde pública e privadas do país.

Assim, necessita-se de um enfoque mais significativo para que possam ser identificadas as possíveis relações com estes construtos e suas causas, para que estas pessoas 
sejam identificadas, encaminhadas e submetidas ao devido tratamento, tendo acompanhamento psicológico/terapêutico/educacional, trazendo assim resultados positivos e, desta forma, protegendo e prevenindo possíveis comportamentos autodestrutivos, como mutilações, suicídio e outros.

Há uma real e significativa necessidade de se realizarem novos estudos sobre autoestima, autoimagem, resiliência e felicidade entre adolescentes escolares, proporcionando uma aproximação, uma compreensão da forma como estas pessoas lidam com as situações diárias de diversidade, que tem origem nos mais diversos campos, dando destaque ao ambiente escolar. As situações que podem proporcionar fatores considerados de risco, como uma possível depressão, que embora não tenha sido abordada neste estudo, pode vir a relacionar-se com os construtos estudados de maneira significativa, desenrolando uma importante e vantajosa aprendizagem quando lidarem com pessoas que enfrentam estes problemas/risco de maneira positiva.

Sugere-se um trabalho visando à conscientização da população, assim como dos profissionais das áreas da saúde, educação e instituições escolares públicas e privadas, sobre os reais riscos com que os adolescentes se deparam diariamente, relacionados aos construtos estudados e, desta forma, contribuir de forma positiva e significativa para que se obtenha um melhor entendimento sobre estes fatores, como se posicionar frente a esta realidade que vem afetando e causando prejuízos à vida dos adolescentes e de todos que estão próximos, não podendo fechar os olhos para as situações adversas e as pessoas envolvidas nelas. Mesmo proporcionando diversos estímulos, é necessário acender a chama do interesse de novos pesquisadores, relacionado a estes temas, por existirem diversas lacunas ainda não preenchidas.

Por fim, tendo entendido que as estimativas relacionadas à forma negativa como estes construtos vêm influenciando os adolescentes que ainda estão na escola aumenta a cada dia, desta forma sendo e tornando-se indispensável a abordagem destes temas/assuntos em futuros estudos. Relativo à resiliência, pode-se destacar os reais avanços que vêm contribuir com a área da saúde. Já relacionado à Autoestima, Autoimagem e Felicidade, faz-se urgente um maior empenho por parte dos pesquisadores nos estudos destes elementos psicológicos, bastante estudados fora do Brasil, de maneira individual e relacionado a outros fatos psicológicos e nas mais diversas áreas. 


\section{REFERÊNCIAS}

LOPES, V. R; MARTINS, M. C. F. Validação fatorial da escala de resiliência de ConnorDavidson (Cd-Risc-10) para brasileiros. Revista Psicologia: Organizações e Trabalho, v. 11, n. 2, p. 36-50, 2011.

PAIS-RIBEIRO, J. L. Validação transcultural da escala de felicidade subjectiva de Lyubomirsky e Lepper. Psicologia, saúde e doenças, v. 13, n. 2, p. 157-168, 2012.

SBICIGO, J. B; BANDEIRA, D. R; DELL'AGLIO, D. D. Escala de Autoestima de Rosenberg (EAR): validade fatorial e consistência interna. Psico-usf, v. 15, p. 395-403, 2010.

GOUVEIA, V. V.; SINGELIS, T. M.; DE MIRANDA, C. J. A. P. Escala de auto-imagem: comprovação da sua estrutura fatorial. Avaliaçao Psicologica: Interamerican Journal of Psychological Assessment, v. 1, n. 1, p. 49-59, 2002.

GRANOT, Y; TYLER, T. R.; DURKIN, A. Legal socialization during adolescence: The emerging role of school resource officers. Journal of Social Issues, 2021.

MOSELLI, M et al. Suicidality and personality pathology in adolescence: A systematic review. Child Psychiatry \& Human Development, p. 1-22, 2021.

MAY, N. N. "I Read the Inscriptions from before the Flood..." Neo-Sumerian Influences in Ashurbanipal's Royal Self-Image. In: Time and History in the Ancient Near East. Penn State University Press, 2021. p. 199-210.

RUBIO, F. (Ed.). Self-esteem and foreign language learning. Cambridge Scholars Publishing, 2021.

MASTEN, A. S.; REED, M. G. J. Resilience in development. Handbook of positive psychology, v. 74, p. 88, 2002.

KATSUMI, Y. et al. Intrinsic functional network contributions to the relationship between trait empathy and subjective happiness. NeuroImage, v. 227, p. 117650, 2021.

QUINTERO, V; ZÁRATE, N. Autoestima, autoconcepto y su relación con el rendimiento académico en estudiantes de odontología. Revista de sistemas y gestión educativa, v. 3, n. 9, p. 11-19, 2016.

MOSQUERA, J. J. M; STOBÄUS, C. D. Auto-imagem, auto-estima e auto-realização: qualidade de vida na universidade. Psicologia, saúde e doenças, v. 7, n. 1, p. 83-88, 2006.

HUA, X. et al. A fast self-attention cascaded network for object detection in large scene remote sensing images. Applied Soft Computing, v. 94, p. 106495, 2020.

TARRÍO, A. M. S. La inscripción latina del túmulo de Luís da Silveira: una auto-imagen sub specie aeternitatis. Documenta \& Instrumenta, n. 19, p. 247-266, 2021. 
BARRAZA MACÍAS, A. Escala de Felicidad Subjetiva: Análisis de sus propiedades psicométricas. Psychologia. Avances de la Disciplina, v. 14, n. 1, p. 29-39, 2020.

RUBIO, F. (Ed.). Self-esteem and foreign language learning. Cambridge Scholars Publishing, 2021.

SAWYER, S. M. et al. The age of adolescence. The Lancet Child \& Adolescent Health, v. 2, n. 3, p. 223-228, 2018.

BLAKEMORE, S. J. Adolescence and mental health. The lancet, v. 393, n. 10185, p. 20302031, 2019.

\section{Como Referenciar este Artigo, conforme ABNT:}

SOUSA, F. F; ROCHA, R. A. S. S. Descrição dos Níveis de Autoestima, Autoimagem, Resiliência e Felicidade de Estudantes Adolescentes. Rev. Saúde em Foco, Teresina, v. 8, n. 2, art. 1, p. 03-14, mai./ago.2021.

\begin{tabular}{|l|c|c|}
\hline \multicolumn{1}{|c|}{ Contribuição dos Autores } & F. F. Sousa & $\begin{array}{l}\text { R. A. S. S. } \\
\text { Rocha }\end{array}$ \\
\hline 1) concepção e planejamento. & $\mathrm{X}$ & \\
\hline 2) análise e interpretação dos dados. & $\mathrm{X}$ & $\mathrm{X}$ \\
\hline 3) elaboração do rascunho ou na revisão crítica do conteúdo. & $\mathrm{X}$ & $\mathrm{X}$ \\
\hline 4) participação na aprovação da versão final do manuscrito. & $\mathrm{X}$ & $\mathrm{X}$ \\
\hline
\end{tabular}

